\title{
Review of: "FAIR RDM (Research Data Management): Italian initiatives towards EOSC implementation"
}

\author{
Silvio Peroni ${ }^{1}$ \\ 1 University of Bologna
}

Potential competing interests: The author(s) declared that no potential competing interests exist.

\section{Metadata}

Title: FAIR RDM (Research Data Management): Italian initiatives towards EOSC implementation Author: Paola Gargiulo, Paola Galimberti, Anna Maria Tammaro, Antonella Zane

Submitted to: 17th Italian Research Conference on Digital Libraries (IRCDL 2021)

\section{Review}

In this paper, the authors describe the outcomes of a survey that investigates which actions are needed to enable the implementation of the EOSC at the Italian level. In principle, the paper's content could be of interest for the IRCDL community and the Italian Open Science community as a whole. However, the paper presents several flaws, the major of which are discussed as follows.

Introduction

In the introduction, I would have expected a narrative that allows the reader to see the problem addressed in the research, understand the study's aim, and comprehend the reasons behind it - i.e. the "whys" such a study is conducted. Instead, the introduction is just a list of initiatives that discusses, in general terms, how Italy is positioned concerning Open Science, with no explicit mention of the goals the authors want to reach with their work.

Some claims contained there are only stated without providing any justification. For instance, on page 3 , the sentence "Galimberti $[3,4]$ highlights that the current research evaluation process is an obstacle to the openness of data and publication" is not accompanied by a justification of why that is an obstacle.

Some half-line paragraphs on page 2 seem to be headings of subsections instead of proper paragraphs. Also, several links and citations are missing - e.g. a link to the EOSC portal in the first line, a citation to the FAIR article in the fourth line, the link to Plan S at page three (the current link goes to a ROARS article, which is not Plan S).

The introduction should be entirely rewritten to fit appropriately the problem addressed and the reason that justifies this study. 
The three research questions the authors had in mind are listed only on page 4. From a general point of view, they are interesting questions. However, due to their very generic nature, one should need several witnesses to gather enough data to address all of them adequately. Instead, the authors were able to find only five respondents plus two "key informants", which is a tiny number of subjects to answer appropriately to the research questions introduced.

All the questions but one have been set as free-text answers. This is good in general, but some additional questions obliging the subjects to answer using a numeric scale (e.g. a likert scale) would have helped the authors present some of the outcomes quantitatively. Also, even the qualitative data (i.e., respondents' answers) would have been elaborated more, e.g., using grounded theory typical of social science studies. The adoption of such a technique would have allowed the authors to group insights from the questionnaire into general categories that could have been used as the basis for a discussion.

\section{Findings}

Some claims listed here are not supported by any evidence. For instance, the authors say that "[s]trangely, there is no experience on a business plan and sustainability issues". What is that strangeness? Could it be derived from the fact that only five respondent have been involved?

Listing the respondents' specific answers in the paper does not provide overall evidence of a community bur rather only specific points of view on the topics addressed. Is there a common trait across different answers that can characterise a uniform view on a specific aspect of those analysed?

I final remark: I found peculiar not to find a link or, better, a citation to a record in an open data repository (e.g. Zenodo) where to find all the data (i.e. questionnaire plus answers received, appropriately anonymised) discussed in this paper.

\section{Conclusions}

The conclusion's organisation in three distinct subsections is unusual, and I do have an issue in justifying it when each subsection contains only one paragraph each. Also, there is no information about possible future works, e.g. extensions of the analysis presented to involve, for instance, a larger set of subjects.

Summarising, the paper in the present form cannot be accepted for presentation at IRCDL as a full paper, since it would need an extensive rewriting. 